\title{
Variability study of the FBS M giants
}

\author{
K. S. Gigoyan ${ }^{* 1}$ and G. R. Kostandyan ${ }^{\dagger 1}$ \\ ${ }^{1}$ V .A. Ambartsumian Byurakan Astrophysical Observatory, Armenia
}

\begin{abstract}
We study in this paper variability of the late-type M giants found in the First Byurakan Survey (FBS) spectroscopic data base. We used phase dependent light - curves from large sky area variability data bases such as Catalina Sky Survey (CSS) and All-Sky Automated Survey for Supernovae (ASAS-SN). We used also the distance information derived from Gaia EDR3 to construct the Galactic distribution of the M - type giants from the second edition of the FBS Late-Type Stars catalogue including various kinds of long period variables.
\end{abstract}

Keywords: Late - type stars: Astronomical data bases-Variability

\section{Introduction}

The study of variable stars is one of the most popular and dynamic areas of the modern astronomical research. Variability is a property of most stars, and as such it has a great deal to contribute to our understanding of them. It provides researchers with many additional and important parameters (periods, amplitudes, etc.) which are not available for non - variable stars. These important physical parameters can be used to deduce characteristics of the stars. The study of variability also allows us to directly observe changes in the stars: both the rapid and sometimes violent changes associated especially with stellar birth and death, and also the slow changes associated with normal stellar evolution. An overview of variable stars, including an introduction to variable stars in general, the techniques for discovering and many-sided studying variable stars, and description of the main types of variable stars are presented in more detail in the book by Percy (2007).

Determination of the correct classes of variables can be very important for studies of stellar populations. Some types of variable stars, such as RR Lyrae stars and Cepheids, are an excellent tool for studying our Galaxy. Being nearly standard candles ( as distance indicators ) and being intrinsically bright, they are a particularly suitable tracer of Galactic structure (Sesar et al., 2007, Tammann et al., 2003, 2008). Long period variables ( LPV, $\Delta \mathrm{V}>2.5 \mathrm{mag}$., or Miras ), which are Asymptotic Giant Branch (AGB) stars, are also very important distance indicators for old and intermediate age populations (Whitelock et al., 2008).

The First Byurakan Spectral Sky Survey (henceforth FBS) low-resolution spectral plates have been used long period to search and study faint late $\mathrm{M}$ - type and C -type (carbob) stars (Late-Type Stars, LTSs) at high galactic latitudes. This objective-prism survey was carried out by Markarian and associates (Markarian et al., 1989) over the 1965 - 1980 period using the $1 \mathrm{~m}$ Schmidt telescope of the Byurakan Astrophysical Observatory (BAO, Armenia). The FBS survey covers total of $17000 \mathrm{deg}^{2}$ segmented in 24 parallel zones (see (Markarian et al., 1989)). Low-resolution photographic plates were obtained using $1.5^{\circ}$ objective-prism giving a reciprocal dispersion of $1800 \AA / \mathrm{mm}$ near $\mathrm{H} \gamma$ throughout a useful field of $4^{o} \times 4^{o}$. During the observations, various Kodak emulsions were used ( IIF, IIAF, IIaF and $103 \mathrm{aF}$ ) providing a $3400-6900 \AA$ spectral range with a wide gap at $5300 \AA$. The FBS original goal was to identify objects with strong UV-excess in a region set by $\delta>-15^{\circ}$ and $\mathrm{IbI}>15^{\circ}$.

\footnotetext{
*kgigoyan@bao.sci.am

†kgayane@gmail.com
} 
All FBS plates have been digitized, resulting in the creation of the Digitized First Byurakan Survey (DFBS) data base (Mickaelian et al., 2007). Its images and spectra are available on the DFBS web portal in Trieste (Italy)(accessed via https:// ia2-byurakan.oats.inaf.it/). All DFBS plates $(\sim 2000)$ were analyzed for LTSs. The second version of the "Revised And Updated Catalogue of the First Byurakan Survey of Late-Type Stars", containing data for 1471 M -and C-stars ( 130 C-type stars, $241 \mathrm{M}$ dwarfs, and $1100 \mathrm{M}$-type giants ) was generated (Gigoyan et al., 2019). Moderate-resolution CCD spectra were obtained for a large fraction of the FBS LTSs at different epochs and with various telescopes (Gigoyan et al., 2019).

\section{Variability study of the FBS M giants}

To determine the variability types of the FBS M - giants, we exploit data from two primary sources, namely the Catalina Sky Survey ( CSS, second public data release CSDR2, accessed via http://nesssi.cacr.caltech.edu/DataRelease/) and the All - Sky Automated Survey for Supernovae ( ASAS-SN, accessed via https://asas-sn.osu.edu/variables/, Jayasinghe et al. (2018), Kochanek et al. (2017), Shappee et al. (2014)). The CSS comprises two main parts surveying the Northern (Drake et al., 2014) and Southern (Drake et al., 2017) sky, respectively. Both surveys were analyzed by the Catalina Real-Type Transient Survey (CRTS) in search for optical transient ( $\mathrm{V}<21.5$ mag. ) phenomena. The ASAS-SN project is an all-sky optical monitoring to a photometric depth $\mathrm{V} \leq 17.0$ mag. providing variability classification. Consequently, ASAS-SN was used as the primary source for attributing variability types, periods, and amplitudes to the FBS M-giants. For the some amounts of the objects missing in the ASAS-SN data base, variability parameters were determined from CSDR2 light curves using the VStar - data visualization and analysis tool (online at http://www.aavso.org/, Benn (2012)).

Our final sample consists of $690 \mathrm{SR}$ ( Semi - Regular ) - type, $300 \mathrm{~L}$ - type ( irregular ) and 110 Mira - type variables.

In Figure1 presented ASAS-SN phase dependence light curves for objects FBS 1808+313 and FBS 2023+029, classified consequently as a Mira -type and Semi-Regular (SR) variables.

Figure 2 presents CSS phase dependence light curve for M giant FBS 2349+181.

Figure 3 presents the distribution of periods for 110 FBS M - type Mira - variables.

Figure 4 present Galactic distribution of the FBS M giants. We used the distance information from early installment of the third Gaia data release ( Gaia EDR3 ) by Bailer-Jones et al. (2021), CDS VizieR Catalogue I/352/gedr3dis).

\section{Discussion and conclusion}

In this paper we explored the sample of $1100 \mathrm{M}$-giants from the second edition of the FBS LTS catalogue various kind of long period variables. For this study, we cross-correlated our sample with the Catalina Sky Survey (CSS) and All-Sky Automated Survey for Supernovae ( ASAS-SN ). These two large sky - area data bases allowed to characterize more detail the variability nature of $\mathrm{M}$ giants. Most of our FBS M - giants are found at typical distances of $1 \mathrm{kpc}$ above or below the Galactic plane. No difference between Miras and SRVs has been found. Many-sided investigations based on modern optical and all-sky infrared astronomical data bases will allow to construct the spectral energy distribution ( SED ) and estimate important physical parameters for them. An analysis of the multiwavelength properties is now being carried out and the results will appear soon for all FBS M giants.

\section{References}

Bailer-Jones C. A. L., Rybizki J., Fouesneau M., Demleitner M., Andrae R., 2021, , 161, 147

Benn D., 2012, , 40, 852 
Drake A. J., et al., 2014, , 213, 9

Drake A. J., et al., 2017, , 469, 3688

Gigoyan K. S., Mickaelian A. M., Kostandyan G. R., 2019, MNRAS, 489, 2030

Jayasinghe T., et al., 2018, , 477, 3145

Kochanek C. S., et al., 2017, , 129, 104502

Markarian B. E., Lipovetsky V. A., Stepanian J. A., Erastova L. K., Shapovalova A. I., 1989, Soobshcheniya Spetsial'noj Astrofizicheskoj Observatorii, 62, 5

Mickaelian A. M., et al., 2007, , 464, 1177

Percy J., 2007, Understanding Variable Stars. Cambridge University Press, https://books.google. am/books? id=GQzCDQI3YP4C

Sesar B., et al., 2007, , 134, 2236

Shappee B. J., Prieto J. L., Grupe D., Kochanek C. S., Stanek K. Z.and Walker Z., Yoon Y., 2014, , 788,48

Tammann G. A., Sandage A., Reindl B., 2003, , 404, 423

Tammann G. A., Sandage A., Reindl B., 2008, , 679, 52

Whitelock P. A., Feast M. W., Van Leeuwen F., 2008, , 386, 313 

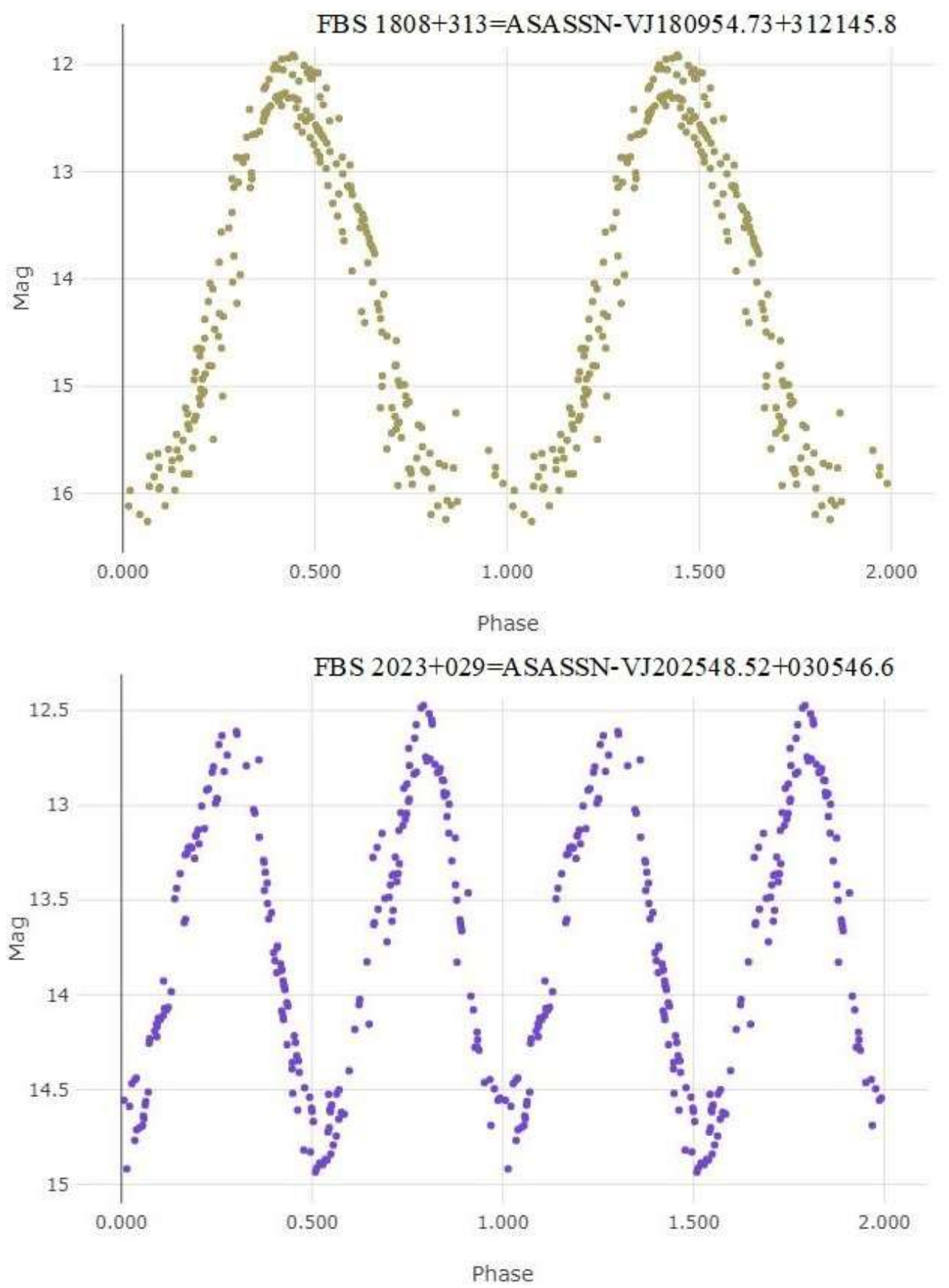

Figure 1. Phased ASAS-SN light curves for two M -giants, FBS 1808+313=ASASSNVJ180954.73+312145.8, classified as a Mira-type variable (Vmean = 13.98 mag., ampl. = 3,82 mag., $\mathrm{P}$ $=240.422$ days) and for FBS 2023+029=ASAS-SN-VJ202548.52+030546.6, classified as a SR variable ( Vmean $=13.74$ mag., ampl.=2.21 mag., $\mathrm{P}=619.654$ days $)$. 


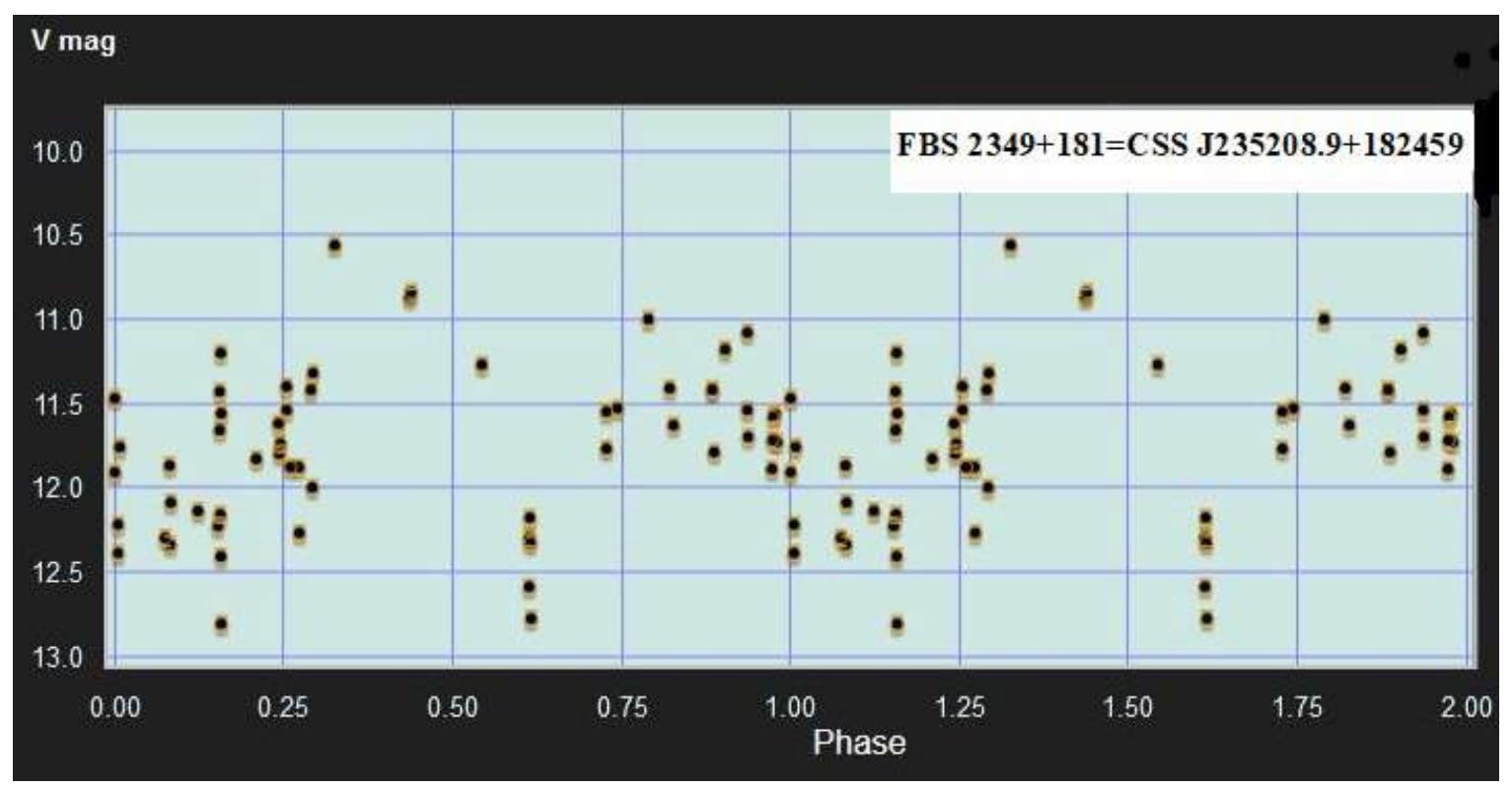

Figure 2. CSS phase dependence light curve for FBS 2349+181=CSS J235208.0+182459. This object classified as a irregular ( L ) variable.

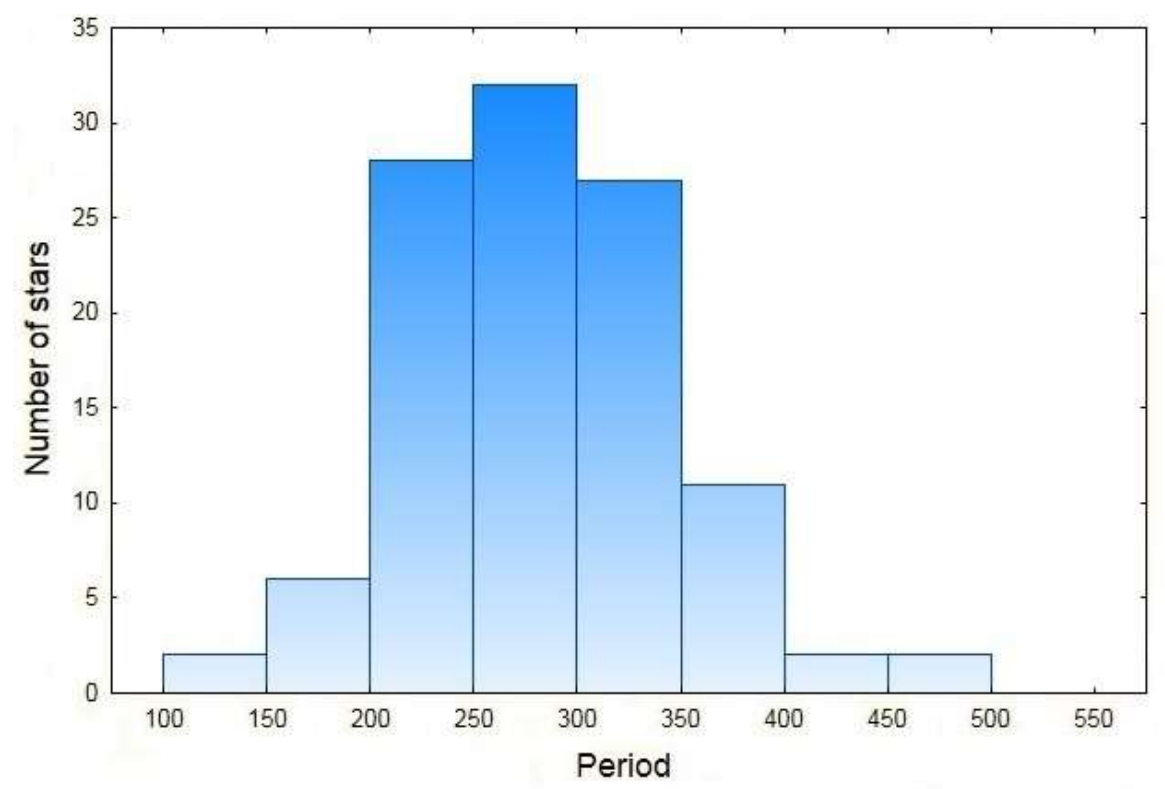

Figure 3. Period distribution of the 110 FBS M - type Mira variables based on ASAS-SN periods. 


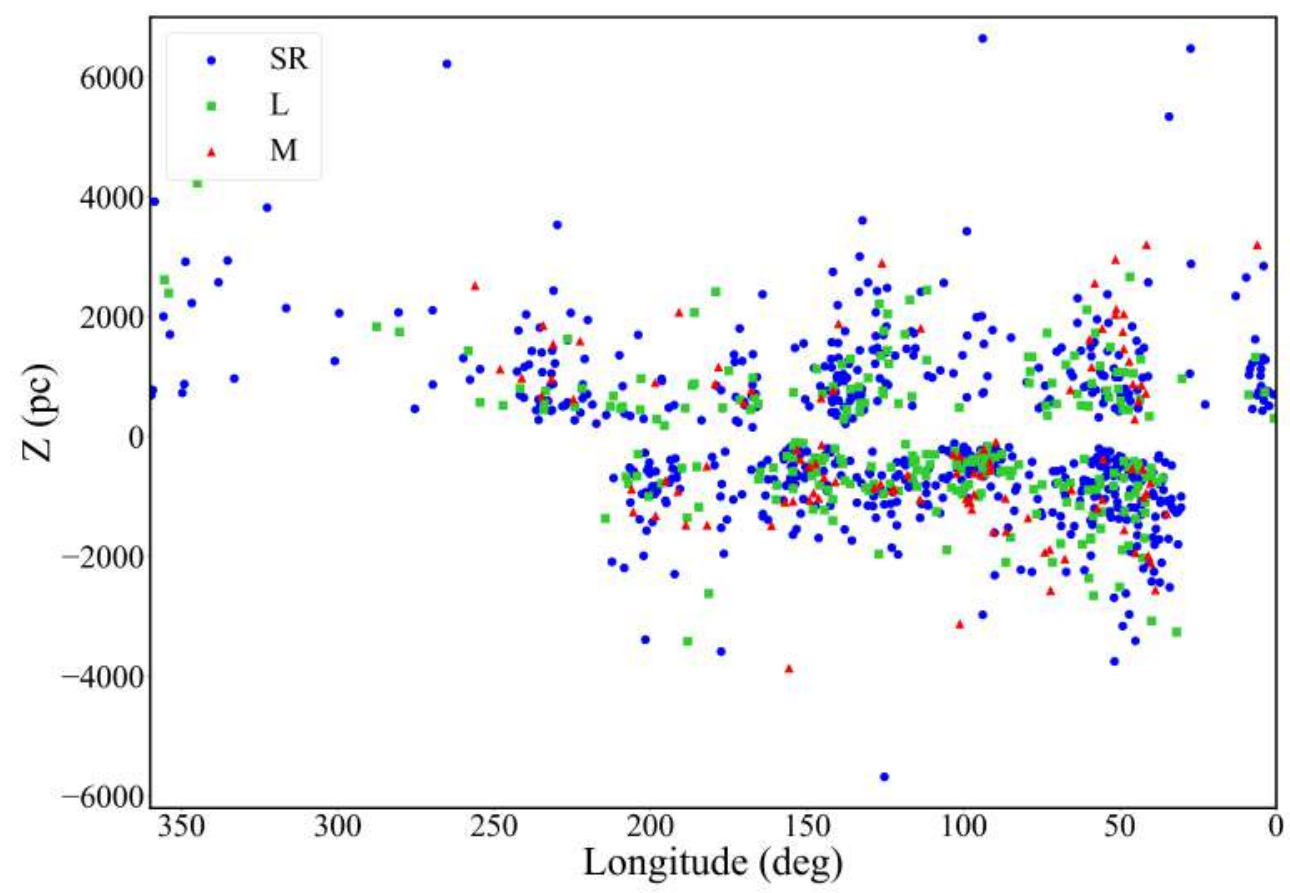

Figure 4. Galactic distribution of the FBS M giants. Symbols are: filled blue squares-SR-variables, filled green squares-irregular ( L ) variables, and red triangles-Mira-type variables. . 